\title{
FLUTTER MECHANISMS IN LOW PRESSURE TURBINE BLADES
}

\author{
M. Nowinski \\ Swiss Federal Institute of Technology \\ EPFL-LTT \\ Lausanne, Switzerland
}

\author{
J. Panovsky \\ GE Aircraft Engines \\ Cincinnati, Ohio USA
}

\begin{abstract}
The work described in this paper is part of a comprehensive research effort aimed at eliminating the occurrence of low pressure turbine blade flutter in aircraft engines. The results of fundamental unsteady aerodynamic experiments conducted in an annular cascade are studied in order to improve the overall understanding of the flutter mechanism and to identify the key flutter parameters. In addition to the standard traveling wave tests, several other unique experiments are described. The influence coefficient technique is experimentally verified for this class of blades. The beneficial stabilizing effect of mistuning is also directly demonstrated. Finally, the key design parameters for flutter in low pressure turbine blades are identified. In addition to the experimental effort, correlating analyses utilizing linearized Euler methods demonstrate that these computational techniques are adequate to predict turbine flutter.
\end{abstract}

\section{NOMENCLATURE}

$c \quad$ chord length

$C_{p} \quad$ coefficient of unsteady pressure, $\tilde{p} / \alpha\left(p_{t 1}-p_{1}\right)$

$C F D \quad$ computational fluid dynamics

$i \quad$ inlet incidence angle

IBPA interblade phase angle

$k$

$L P T$

$M_{1}$

$\stackrel{M}{2}_{2}$

$\tilde{p}$

$P S$

$p_{t 1}$

$p_{1}$

$s$

SS

$U_{2}$

$\alpha$

$\omega$ reduced frequency, $\omega c / 2 U_{2}$

low pressure turbine

inlet Mach number

outlet Mach number

unsteady pressure

pressure side of the blade

inlet total pressure

inlet static pressure

normalized blade surface distance

suction side of the blade

outlet velocity

blade angular displacement

blade vibration angular frequency

\section{INTRODUCTION}

This paper summarizes the results of a series of experiments conducted in an annular cascade facility to investigate torsional flutter in low pressure turbine (LPT) blades. The development of improved design guidelines for LPT flutter is an active research area because there have been several recent occurrences of instability in this class of blades. Because of these concerns, a research project was initiated with the goal of eliminating flutter in LPT blades.

To date, several forced vibration experiments similar to those described in this paper have been conducted in both linear and annular cascades (Bölcs and Schläfli, 1984, Buffum and Fleeter, 1990). In general, this work has been focused on a characterization of the aerodynamic stability of the cascade based on measurements of the blade surface unsteady pressures. For the current LPT cascade, this topic was the general focus of a previous paper (Panovsky, Nowinski, and Bölcs, 1997). In addition, comparisons were presented between the measured unsteady pressures and predictions from computational fluid dynamics (CFD) codes.

The current paper represents an extension of this work. The primary objective of the tests presented here is to investigate the influence of the reduced frequency, inlet flow incidence, location of the torsion axis, and other key parameters on the cascade unsteady response. In addition, an enhanced understanding of the unsteady behavior of the cascade is obtained based on unsteady pressure measurements made along the test section outer wall. Tests were also performed to check the applicability of the influence coefficient technique to these cases, as well as to study the effects of cascade mistuning. Computational predictions are included to compare with the experimental measurements.

\section{EXPERIMENTAL FACILITY AND DATA ANALYSIS}

In this section, a brief description of the experimental facility, test article, and data analysis technique is provided. A more complete presentation can be found in Panovsky, Nowinski, and Bölcs (1997). The experimental measurements were conducted in the non-rotating annular test facility (Bölcs, 1983) at the Ecole Polytechnique Fédérale 
de Lausanne (EPFL). This is a continuous flow, open cycle facility with a test section measuring $160 \mathrm{~mm}$ (inner radius) by $200 \mathrm{~mm}$ (outer radius). The flow conditions at the test section inlet can be varied over a wide range of Mach numbers ( 0.3 to 1.6$)$ and flow angles $\left(20^{\circ}\right.$ to $70^{\circ}$ ). The general intent of the facility is to provide two-dimensional flow conditions. In addition, it provides important advantages for oscillating blade measurements due to its inherent circumferential flow periodicity and absence of lateral boundaries.

The test cascade is composed of an annular arrangement of twenty blades. The LPT blade shape used for this series of tests corresponds to a latter stage of a modern commercial turbofan engine. Each airfoil in the cascade is imbedded within a base whose curved surface also forms the inner boundary of the test section. Each base is attached to its own mass and spring element, and the resulting blade assemblies are mounted to a common core.

The individual blades in the cascade are excited using an array of electromagnets. Control of the vibration amplitude and interblade phase angle (IBPA) is accomplished electronically based on feedback signals provided by inductive displacement transducers placed over each individual blade assembly (Kirschner, Fosco, and Muller, 1980). The frequency and mode shape are based on the torsional mode of the actual blade. To permit the investigation of the effects of changes in the mode shape, provisions for three locations of the torsion axis were made. These locations are shown in the blade schematic of Figure 1. The nominal blade vibration amplitude is approximately $0.5^{\circ}$.

The upstream and downstream steady-state flow conditions are determined from spanwise-circumferential traverse data obtained using 3-hole aerodynamic probes. In addition, throughflow information is provided by a series of static pressure taps located along the outer wall of the test section. A parallel line of taps are available for unsteady pressure measurements. Two neighboring blades in the cascade are also instrumented with a total of 18 static pressure taps at midspan to measure the steady blade surface pressure distribution. A second pair of blades are instrumented with 18 miniature piezoresistive pressure transducers at midspan to measure the unsteady blade surface pressures. These measurement locations are also shown in Figure 1.

The raw, time-dependent data derived from the pressure transducers and the inductive displacement transducers is composed of small amplitude, continuous voltage signals. These analog signals are treated through a chain of filters and amplifiers, and subsequently digitized and stored. The data sample is then subdivided into blocks, and an FFT decomposition, including the application of a data window to reduce leakage effects, is performed on each block. The frequency component corresponding to the blade vibration reference frequency is extracted from each data block, and an average complex value is calculated. The transducer sensitivities, and any other calibration corrections are applied to these average values. The standard deviations for each data set are calculated using the average and individual block values. The $95 \%$ confidence intervals are calculated from the standard deviation estimates assuming a Student's tdistribution. For these measurements, typical 95\% confidence intervals are on the order of $\pm 3 \%$ for the unsteady pressure amplitude, and $\pm 4^{\circ}$ for the unsteady pressure phase angle.

\section{COMPUTATIONAL PROGRAM}

Computational results using a quasi-3D linearized Euler code called NOVAK (Holmes and Chuang, 1993) have been obtained for all of the experimental cases. These predictions are compared to the experimental results in terms of the unsteady pressure, work, and damping coefficients (Bölcs and Fransson, 1986). All presented calculations were performed without streamtube contraction, which was found to only slightly affect the unsteady predictions (Panovsky, 1997). The primary purpose of the analytical investigations is for comparison with the experimental measurements to determine whether inviscid methods are applicable to LPT flutter.

In linearized Euler methods, the unsteadiness in the flowfield is assumed to be a small harmonic perturbation to the nonlinear steady flow governed by the Euler equations. The linearized Euler approaches seem to be the best compromise of accuracy and efficiency available at the present time. These methods can include the effects of the geometry of the blades, and model the most pertinent physics of the flowfield. Shocks can be directly captured by the steady solutions, and their effects on the unsteadiness determined. Because of the assumptions inherent in the linearization, variations in the flow parameters are limited to small harmonic perturbations. The determination of incipient flutter meets this requirement, since actual LPT blades vibrate at very small amplitudes. Obviously, Euler methods cannot model the boundary layers and other viscous regions accurately. The numerical solutions for blades operating at high incidence angles, as sometimes required of LPT blades, may not be accurate.

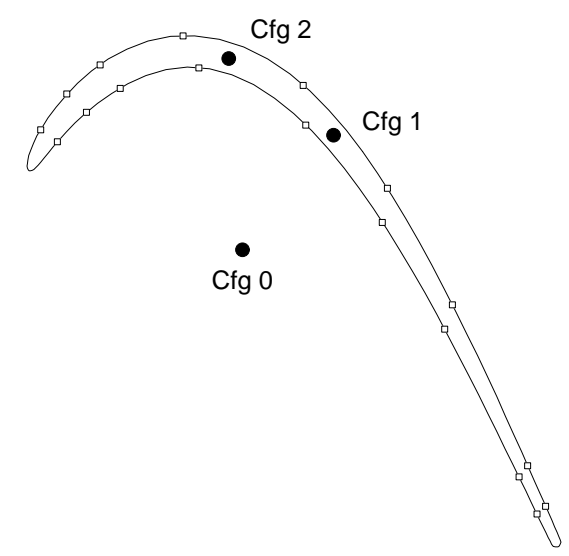

Figure 1. Blade Profile with torsion axes and transducer locations

\section{RESULTS \\ Description of Test Cases}

Measurements for four to five steady flow conditions were conducted for each of the three torsion axis locations mentioned previously. The most significant parameters are summarized in Table 1. The sets of tests corresponding to each of the torsion axis locations will be collectively referred to as "Configurations". The range of steady conditions shown reflects primarily variations to incidence angle, although changes to other steady flow parameters were also addressed.

Three different types of unsteady experiments were conducted. The first is the traveling wave mode, where each blade is driven to a constant amplitude and there is a constant temporal phase difference between adjacent blades. This mode simulates the dominant characteristics of the system mode behavior of the actual rotor assembly (Lane, 1956). A total of twelve interblade phase angles are used for each of the steady conditions. 
The second type of experiment will be called the single blade vibration test, where each blade in the cascade is vibrated individually. Measuring the unsteady pressures generated on the instrumented (reference) blade while vibrating single blades in the cascade gives a direct determination of the influence coefficients (Crawley, 1988). These coefficients define the relative importance of each blade in the cascade to the generation of unsteady pressures on a given reference blade.

The final type of experiment is referred to as the alternating blade test, where only every other blade in the cascade is excited in a traveling wave mode. This test is a simulation of mistuning, which studies the effect of the small frequency variations of actual blades on the overall vibratory response of the rotor. The driven blades model those that would be at a system mode natural frequency while the undriven blades simulate those that are detuned from the system frequency. The arrangement selected is considered the most stable mistuning pattern, at least from a robust design viewpoint (Crawley and Hall, 1985).

Table 1. Summary of test cases

\begin{tabular}{|c|c|c|c|c|c|}
\hline Cfg. \# & Cond. \# & $\mathrm{M}_{1}$ & $\mathrm{M}_{2}$ & $\mathrm{k}$ & $\mathrm{i}\left({ }^{\circ}\right)$ \\
\hline 0 & 1 & 0.37 & 0.74 & 0.31 & 0.7 \\
\hline 0 & 2 & 0.50 & 1.00 & 0.24 & 1.1 \\
\hline 0 & 3 & 0.46 & 0.95 & 0.25 & 4.9 \\
\hline 0 & 4 & 0.47 & 0.83 & 0.28 & 10.4 \\
\hline 1 & 1 & 0.38 & 0.77 & 0.16 & 0.6 \\
\hline 1 & 2 & 0.50 & 0.76 & 0.17 & 6.3 \\
\hline 1 & 3 & 0.46 & 0.77 & 0.16 & 3.6 \\
\hline 1 & 4 & 0.47 & 0.67 & 0.19 & 9.3 \\
\hline 2 & 1 & 0.39 & 0.66 & 0.18 & 0.4 \\
\hline 2 & 2 & 0.49 & 0.68 & 0.17 & 5.8 \\
\hline 2 & 3 & 0.46 & 0.54 & 0.21 & 5.4 \\
\hline 2 & 4 & 0.36 & 0.45 & 0.25 & 6.2 \\
\hline 2 & 5 & 0.42 & 0.52 & 0.22 & 0.7 \\
\hline
\end{tabular}

\section{Traveling Wave Tests}

A representative case from the traveling wave results, corresponding to the design point conditions (Condition 1) for Configuration 1, is presented in this section. The distribution of the first harmonic of the unsteady pressure measured along the blade surface is given in Figure 2 for two interblade phase angles, IBPA= $-90^{\circ}$ and $\mathrm{IBPA}=+90^{\circ}$. It can be observed from these plots that, in general, the highest amplitude unsteady pressures occur in the leading edge region up to $s=0.5$ on the suction side, and near the trailing edge on the pressure side. Computational results calculated by the NOVAK code are also included in these figures. These results are in reasonably good agreement with the experimental data and, in particular, accurately capture the trends exhibited along the blade surface and the effects of changing IBPA. Some differences are evident, especially in the magnitude of the unsteady pressures.

Additional insight into the influence of IBPA is provided by the contour plots in Figure 3 which show the measured distribution of the unsteady pressure amplitude on the blade surface as a function of IBPA. On both blade surfaces, the unsteady pressure peak regions illustrated in Figure 2 are most prominent near IBPA $=180^{\circ}$, and tend to disappear near IBPA $=0^{\circ}$. This behavior points to the important influence of the direct neighbor blades on the instrumented blade unsteady pressures.
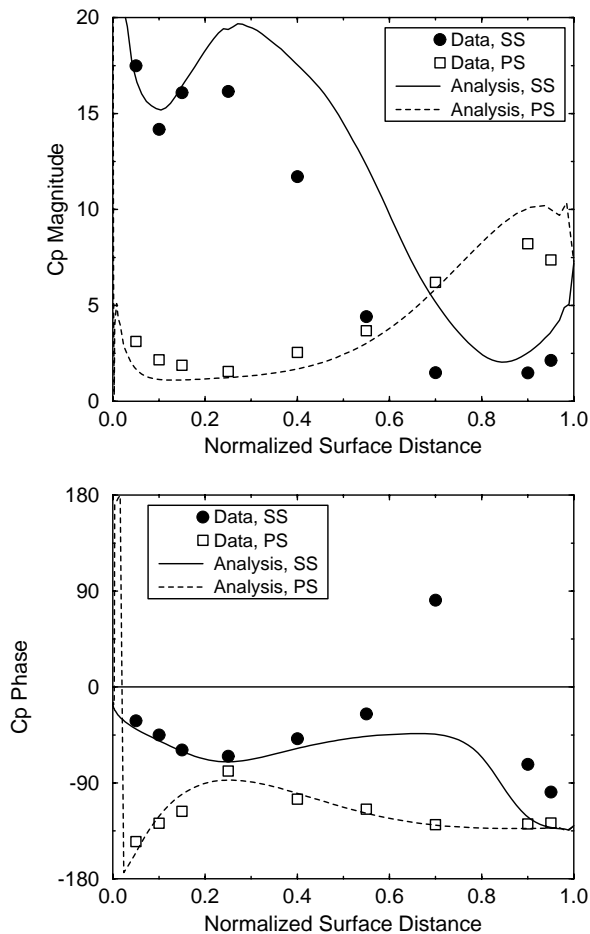

(a)
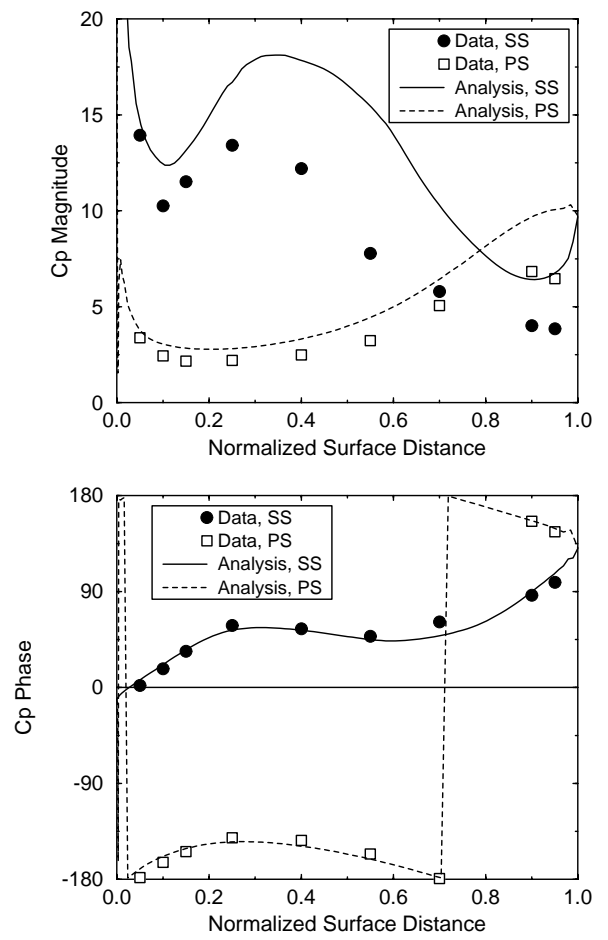

(b)

Figure 2. Magnitude and phase of unsteady pressure: (a) $I B P A=-90^{\circ}$, (b) $I B P A=+90^{\circ}$ 
Further information concerning the interaction of the neighboring blades can be obtained from the unsteady pressure measurements obtained along the outer wall of the test section. Such measurements have been previously presented by Körbächer (1996) and represent a unique opportunity to investigate the unsteady interaction of the blades in the cascade by experimental means. Figure 4 shows the instantaneous pressure distribution within two blade passages for $\mathrm{IBPA}=180^{\circ}$. Three different positions of the blades are shown: (a) largest throat area between the two blades, (b) mean throat area, and (c) smallest throat area. The mean position of the blade is indicated by the black profile, and the instantaneous position is indicated by the white profile. Due to the torsional motion of the blade, the trailing edge experiences the largest linear displacement. This can lead to fairly significant changes in the throat area of the blade passage during cascade vibration, particularly for IBPA $=180^{\circ}$. It can be observed in Figure $4 \mathrm{a}$ that for the largest throat area, the measured unsteady pressures in the center passage are small. Figures $4 \mathrm{~b}$ and $4 \mathrm{c}$ illustrate an increase in the instantaneous unsteady pressure as the two blade trailing edges approach one another. For the smallest throat area, the aforementioned peak unsteady pressure regions along the leading edge on the suction side and along the trailing edge of the pressure side become apparent. It can also be observed from this series of plots that these peak regions are coupled to the same basic unsteady flow phenomenon.

The aerodynamic damping coefficient can be calculated from the unsteady pressure measurements using the standard expression (Bölcs and Fransson, 1986). In Figure 5, the damping distribution along the blade surface as a function of IBPA is shown. The zero damping contour is represented in these plots by a black line. Note that the maximum damping contributions occur near $s=0.25$ on the suction surface and near the trailing edge on both surfaces. The primary destabilizing influences (negative damping) occur near IBPA $=+90^{\circ}$.

The damping distribution can be integrated over the blade surface to obtain the overall damping as a function of IBPA, as shown in Figure 6. This plot indicates there is only a small range of IBPA near $+90^{\circ}$ where the cascade is unstable. Comparison of the test measurements and computational predictions are shown to be in very good agreement. Similar comparisons for all of the steady conditions listed in Table 1 have been conducted, and indicate that linearized Euler methods are sufficient for the prediction of LPT flutter.

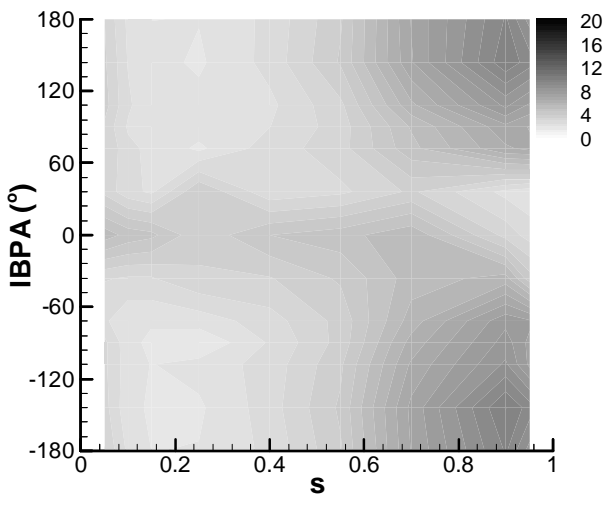

(a)

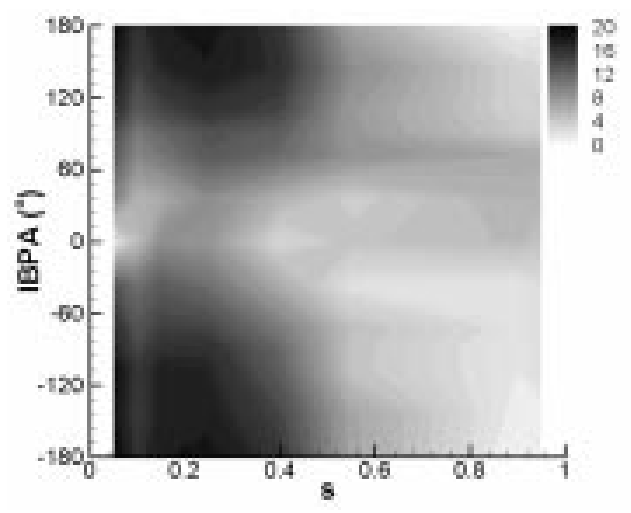

(b)

Figure 3. Measured $C_{p}$ magnitude: (a) $P S$, (b) $S S$

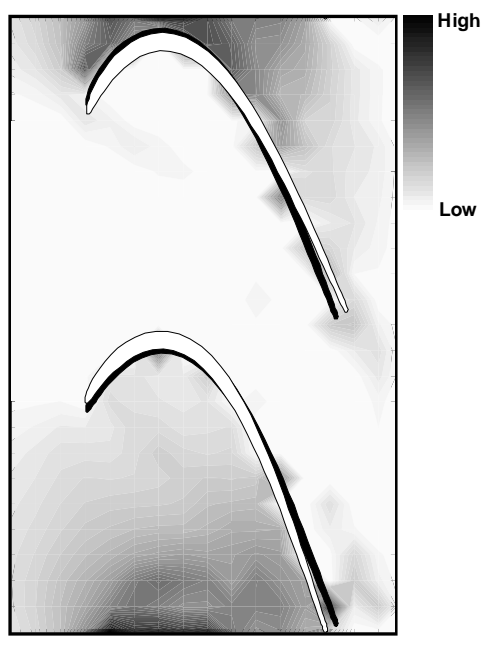

(a)

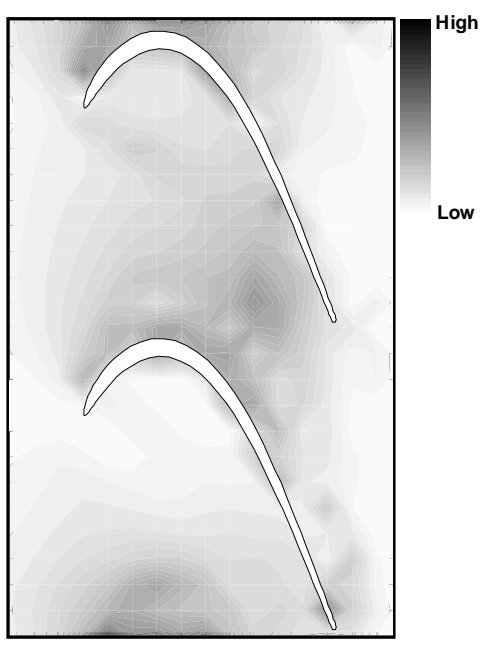

(b)

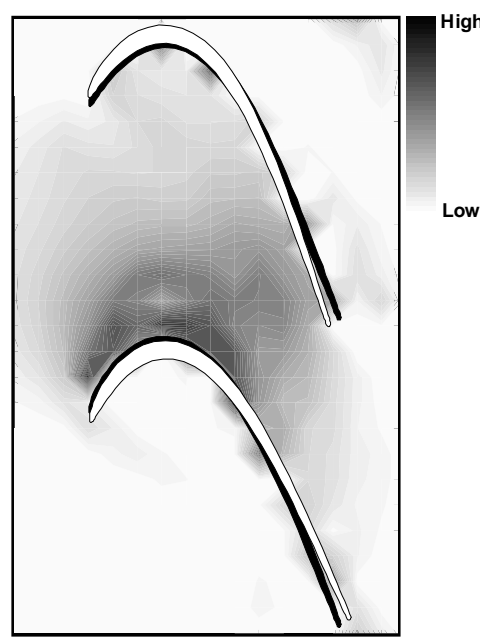

(c)

Figure 4. Instantaneous pressures from outer wall measurements for $I B P A=180^{\circ}$ 


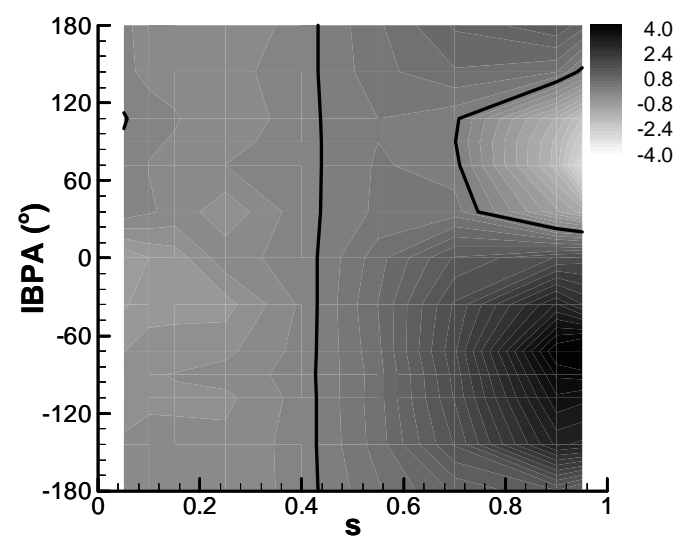

(a)

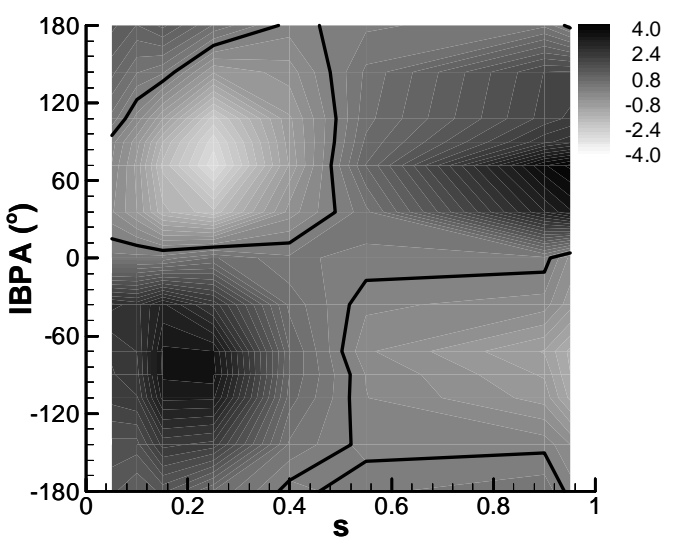

(b)

Figure 5. Experimental damping coefficient distribution:

(a) PS, (b) SS

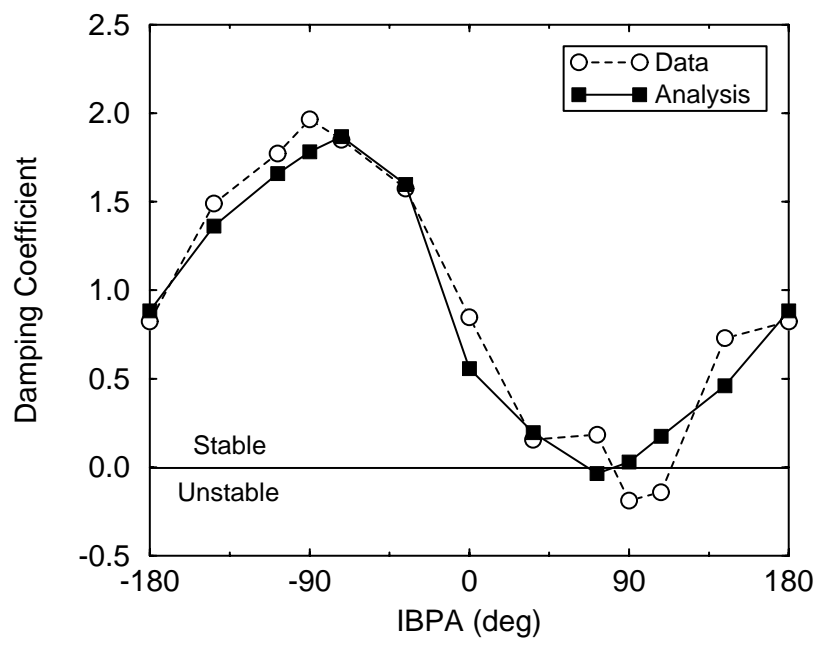

Figure 6. Overall damping coefficient

\section{Single Blade Tests}

The unsteady pressures which occur on a given blade surface represent a combination of the contributions made by the individual vibrating blades in the cascade. These contributions are generally referred to as the influence coefficients. It is also possible to define influence coefficients for local and overall work and damping. The blade numbering convention utilized in this paper defines the reference blade as blade 0 , with the +1 blade immediately to the pressure side, the -1 blade immediately to the suction side, and so on to define the entire cascade.

The contribution from each blade in the test cascade can be directly determined by vibrating only single blades and measuring the unsteady pressures generated on the instrumented (reference) blade. The influence coefficients can also be obtained mathematically from the traveling wave results by assuming a linear superposition of the unsteady pressures in the cascade. By comparing the single blade and traveling wave influence coefficients, the validity of this assumption can be verified. For Configuration 1, Condition 1, the results of this comparison are shown in Figure 7 for a single unsteady pressure transducer located at $s=0.25$ on the suction surface. Both the magnitude and the phase are shown to be in excellent agreement. Other locations and conditions (refer to Table 1), give similar correlations. Thus, the influence coefficient technique is demonstrated to be valid for the case of LPT flutter. Similar linearity validations were reported by Bölcs, Fransson, and Schläfli (1989) and Körbächer and Bölcs (1996) for annular turbine and compressor cascades, respectively, vibrating in the bending mode. However, to the authors' knowledge, this is the first time that the superposition principle has been validated for LPT blades vibrating in torsion. This determination also provides a potential opportunity to greatly simplify the experimental setup, as it implies that the traveling wave results can be accurately simulated by vibrating only a single blade in the cascade and measuring the unsteady pressures on the various neighboring blades.

The influence coefficients also provide important information regarding the relative importance of the motion of individual blades in the cascade to the generation of time-dependent pressures at a given location. In general, for this cascade, only the contributions from the 0 or reference blade and the neighboring +1 and -1 blades have a significant influence on the unsteady pressures. Along the suction surface, the 0 blade exerts the largest influence near the leading edge. The neighboring -1 blade (which faces the suction side of blade 0 ) also exerts a significant influence at this location. Near the mid-section of the blade, the influence of the -1 blade substantially increases, becoming larger than that of the $O$ blade. The important influence of this neighboring blade was cited in the previous section. Near the trailing edge, the influence of the -1 blade diminishes greatly and the largest influences are derived from the 0 and +1 blades. Along the pressure side, the primary influence is due to the 0 blade except near the trailing edge. Here, the influence of the +1 blade (which faces the pressure side of blade 0 ) is also significant. 


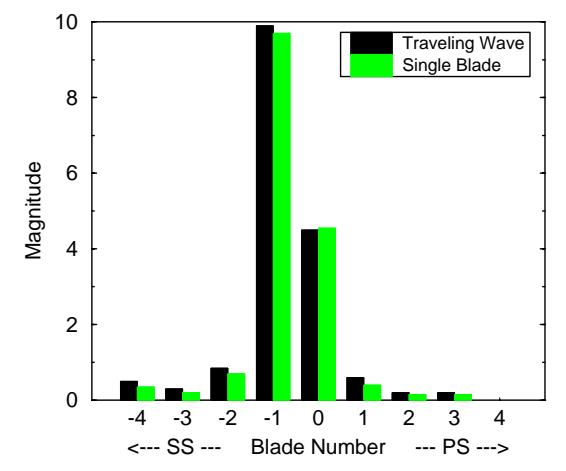

(a)

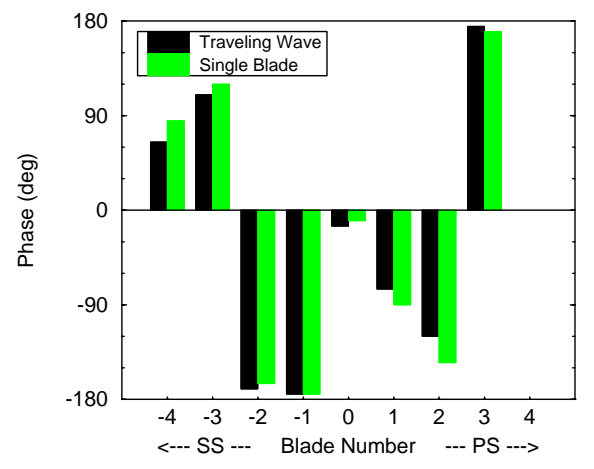

(b)

Figure 7. Comparison of influence coefficients for $s=0.25$ on the SS: (a) Magnitude, (b) Phase

\section{Alternating Blade Tests}

The effects of mistuning were evaluated by vibrating only alternate blades in the cascade in a traveling wave pattern. Unsteady pressures were recorded as for the other tests. It was found that this vibration mode had a powerful stabilizing effect on the cascade, as has been indicated by previous research (Kaza and Kielb, 1982; Crawley and Hall, 1985).

The reason for this stabilizing effect is clearly evident when analyzing the unsteady pressure distribution on the blade. The unsteady pressures near $s=0.25$ on the suction side for IBPA $=+90^{\circ}$ are the most destabilizing on the blade (see Figures 3 and 5). As mentioned previously, this unsteadiness is primarily due to the relative motion of the -1 blade. By not allowing the $-l$ blade to vibrate, this source of unsteadiness is essentially removed, and an improvement in the overall stability of the blade results.

The stabilization can also be explained by a consideration of the influence coefficients of the overall damping from the normal traveling wave experiments. The contribution from the reference blade is the average damping value across the IBPA range. The influence coefficients show that the only significant contribution from other blades is from the immediate neighbors, the -1 and +1 blades. In the traveling wave domain, these contributions will appear as the first harmonic variations with IBPA. If these contributions are removed by fixing these blades, the minimum stability over all IBPA will increase.
Since only the minimum stability is of concern in a practical sense, the stability of the blade improves.

An example of the results of the mistuning experiments is shown in Figure 8 for Configuration 1, Condition 1. The effect of removing the contributions of the neighboring blades is readily apparent, as the damping coefficient over the entire IBPA range approaches the average value. It is worth noting that the unsteady pressures produced by this vibration pattern and, in fact, any arbitrary vibration pattern, can be also accurately determined from the influence coefficients.

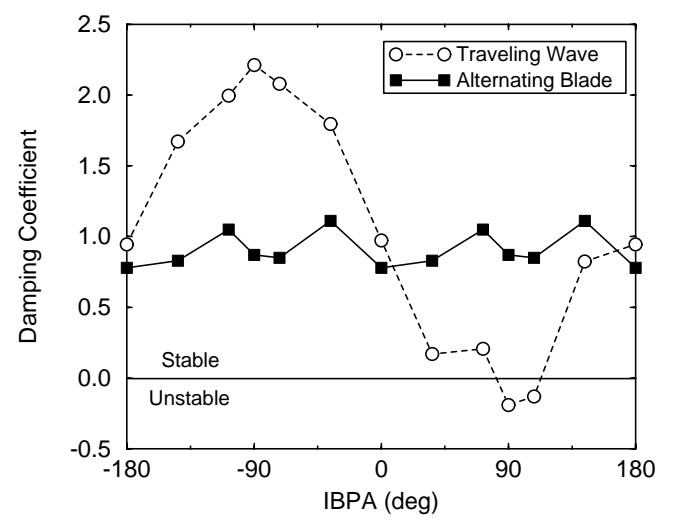

Figure 8. Effect of mistuning on overall damping coefficient

\section{KEY DESIGN PARAMETERS}

We now utilize the experimental results to identify the effect of key design parameters on the stability characteristics of the cascade. To conduct this study, the least stable point from the curve of damping versus IBPA is identified for each of the steady conditions summarized in Table 1. This minimum damping value is the primary value of interest from a stability evaluation standpoint. These damping values were then plotted as a function of every significant steady and unsteady parameter associated with the various steady conditions.

An example is shown in Figure 9 for two of the parameters which are usually considered to be the most important, reduced frequency and incidence. Each steady condition is denoted by a numerical index which corresponds to the Configuration number. Thus, there are four data points for Configurations 0 and 1, while Configuration 2 has five points. Acknowledging that the available data set does not reflect independent variations of these parameters, it is nevertheless surprising that neither of these plots indicates a clear trend in the damping. The primary trend shown is that the conditions are grouped into Configurations, implying that the primary driver is mode shape. If we consider the data points within a particular Configuration, a secondary trend is observed for the reduced frequency. For example, within the five data points for Configuration 2, there is a clear trend toward lower damping as the reduced frequency drops. A dotted line is used to indicate this trend for each of the Configurations. The incidence plot, however, does not exhibit even this secondary trend.

Loading in general, in terms of higher pressure ratio or higher exit Mach number or other similar measure, seems to have a secondary influence, but no single parameter could be identified as having the dominant contribution. In fact, the Zweifel number (Zweifel, 1945), which is the primary loading guideline, shows no trend at all. Examples of the effect of loading are shown in Figure 10 using the ratio of the inlet and exit static pressures and the Zweifel number as 
parameters. Average trends are again indicated by dotted lines in the pressure ratio plot. In summary, this study indicates that the most dominant contribution to overall stability for LPT blades is the mode shape, followed by reduced frequency, and finally some type of loading parameter.

The influence coefficients of the overall stability can also be used to assess the importance of the individual blades to the observed trends. It is found that the mode shape strongly affects contributions from the neighboring -1 and +1 blades. The reduced frequency affects contributions from both the reference blade and the adjacent blade pair. Loading variations solely affect the reference blade. Incidence variations did not seem to have a significant effect on the stability characteristics of the cascade through the range tested. These results are summarized in Table 2. It should be noted that these conclusions have been drawn based on the experimental data only, and mostly serve to highlight the important design parameters. A more detailed parameter study employing computational methods is presented by Panovsky and Kielb (1998).

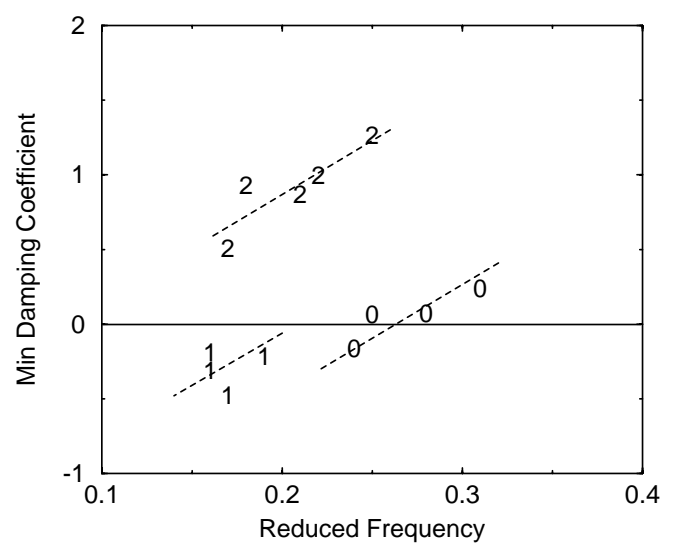

(a)

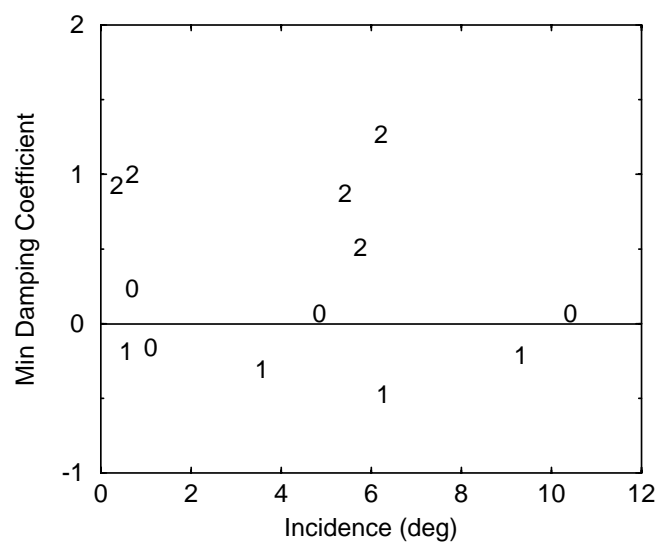

(b)

Figure 9. Trends of minimum damping versus key parameters: (a) reduced frequency, (b) incidence

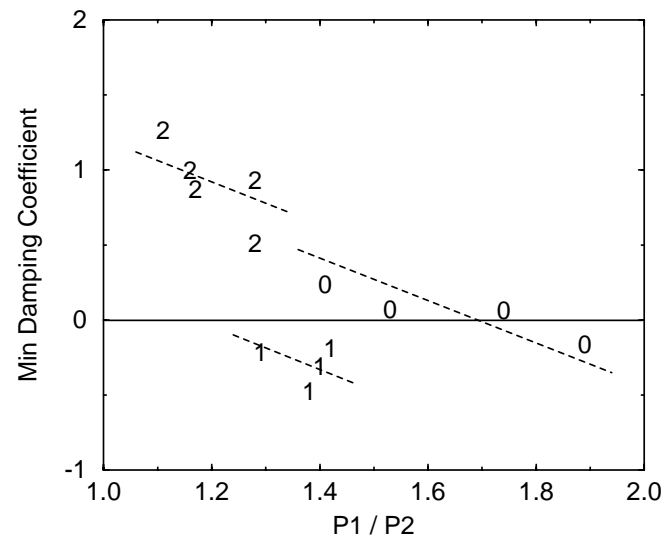

(a)

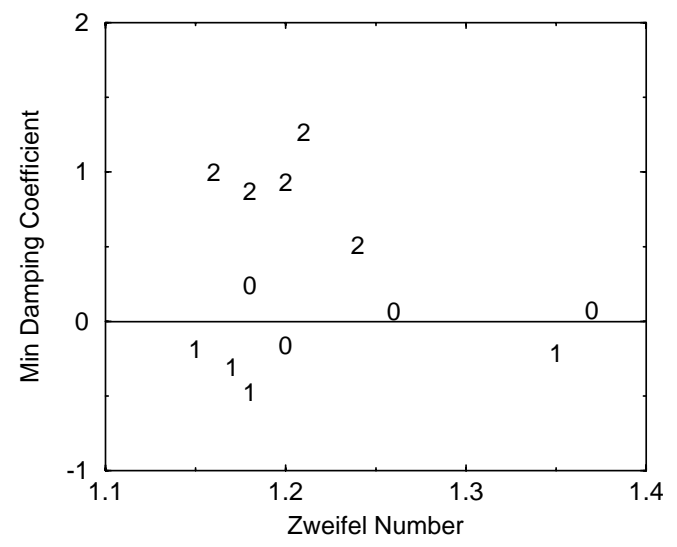

(b)

Figure 10. Trends of minimum damping versus key parameters: (a) static pressure ratio, (b) Zweifel number

Table 2. Dependence of the damping influence coefficients on key parameters

\begin{tabular}{|c|c|c|c|c|}
\hline Blade(s) & Mode Shape & $\boldsymbol{k}$ & Incidence & Loading \\
\hline Reference & secondary & secondary & none & secondary \\
\hline Adjacent pair & primary & secondary & none & none \\
\hline
\end{tabular}

\section{SUMMARY AND CONCLUSIONS}

Detailed measurements of the unsteady pressures generated by the vibratory motion of a modern LPT blade in an annular cascade have been completed. A total of thirteen steady conditions were investigated using three torsion axis locations. Several types of unsteady tests were conducted, including traveling wave, single blade, and alternating blade modes.

The traveling wave results demonstrated that the highest amplitude unsteady pressures were typically generated on the front half of the blade along the suction side, and near the trailing edge on the pressure side. The important role that these regions play in determining the overall stability of the blade was also shown. In addition, using the outer wall unsteady pressure measurements and the blade influence coefficients, the interaction of the direct neighbor 
blades was identified as the primary source for these peak unsteady pressures.

By comparing the influence coefficients from the traveling wave and single blade mode tests, the validity of the linear superposition approach for unsteady pressures was demonstrated for LPT blades. The alternating blade mode tests explicitly demonstrate the benefits of mistuning for flutter. The resulting stabilization is expected based on the work of previous researchers, and the reason for this behavior becomes particularly obvious when considering the important influence on the stability attributed to the adjacent blades, as indicated by the influence coefficients and outer wall measurements.

Correlating analyses for all of the experimental data have been completed using a quasi-3D linearized Euler code. Detailed comparisons of the measured and predicted unsteady pressures and damping coefficients were provided and discussed. The results indicate that this type of method can be used to reliably assess the stability of LPT blades.

Results of the experimental data were used to determine the key parameters for LPT flutter. This investigation indicates that there is a fairly small range of IBPA where flutter can occur for this blade. This range is a function of mode shape, frequency, and steady conditions. However, the most significant factor in determining stability was shown to be the mode shape. While all of the tests conducted used a torsion mode, relatively small changes to the location of the torsion axis had a dramatic effect on the stability behavior. This is an extremely important conclusion because the primary design parameter up to this time has been the reduced frequency. Reduced frequency and loading were also found to have an influence, though to a lesser degree than mode shape. Incidence angle did not have a significant effect on stability over the range tested. The importance of these parameters was also expressed in terms of the corresponding influence coefficients. This led to the conclusion that variations in the reference blade contributions are driven primarily by reduced frequency and loading. The adjacent blade pair contributions vary primarily due to changes in mode shape, though reduced frequency has a secondary effect.

\section{ACKNOWLEDGEMENTS}

The authors wish to thank GE Aircraft Engines and Ecole Polytechnique Fédérale de Lausanne for permission to publish this paper. The first author would like to thank Dr. Peter Ott and Dr. Heiko Körbächer for their valuable technical comments. The second author would like to thank Dr. Graham Holmes, Dr. Christopher Lorence, Dr. Steven Manwaring, and especially Dr. Robert Kielb; thanks are also extended to Prof. Torsten Fransson of the Royal Institute of Technology in Sweden

\section{REFERENCES}

Bölcs, A., "A Test Facility for the Investigation of Steady and Unsteady Transonic Flows in Annular Cascades," ASME Paper 83GT-34, 1983.

Bölcs, A., and Fransson, T.H., "Aeroelasticity in Turbomachines: Comparison of Theoretical and Experimental Cascade Results," Air Force Office of Scientific Research, AFOSR-TR-87-0605, 1986.

Bölcs, A., Fransson, T.H., and Schläfli, D., "Aerodynamic Superposition Principle in Vibrating Turbine Cascades," AGARD $74^{\text {th }}$ Specialists' Meeting of the Propulsion and Energetics Panel on Unsteady Aerodynamic Phenomena inTurbomachines, Luxembourg, August 28-September 1, 1989.
Bölcs, A., and Schläfli, D., "Flutter Phenomena in a Transonic Turbine Cascade," Unsteady Aerodynamics of Turbomachines and Propellers: Proceedings of the Symposium, Cambridge, England, pp. 411-425, September 24-27, 1984.

Buffum, D. H., and Fleeter, S., "Aerodynamics of a Linear Oscillating Cascade," NASA Technical Memorandum 103250, August 1990.

Crawley, E.F., "Aeroelastic Formulation for Tuned and Mistuned Rotors," Chapter 19 in AGARD Manual on Aeroelasticity in AxialFlow Turbomachines, Vol. 2, Structural Dynamics and Aeroelasticity, M.F. Platzer and F.O. Carta, eds., AGARD-AG-298, 1988.

Crawley, E.F., and Hall, K.C., "Optimization and Mechanisms of Mistuning in Cascades," ASME Journal of Engineering for Gas Turbines and Power, Vol. 107, No. 2, pp. 418-426, 1985.

Holmes, D.G., and Chuang, H.A., "2D Linearized Harmonic Euler Flow Analysis for Flutter and Forced Response," in Unsteady Aerodynamics, Aeroacoustics, and Aeroelasticity of Turbomachines and Propellers, H.M Atassi, ed., Springer-Verlag, New York, 1993.

Kaza, K.R.V., and Kielb, R.E., "Flutter and Response of a Mistuned Cascade in Incompressible Flow," AIAA Journal, Vol. 20, No. 8, pp. 1120-1127, Aug., 1982.

Kirschner, A., Fosco, B., and Muller, E., "Control of Vibration in Aeroelastic Experiments," Communication de l'ITA/EPFL, No. 10, pp. 285-295, 1980.

Körbächer, H., "Experimental Investigation of the Unsteady Flow in an Oscillating Annular Compressor Cascade," Ph.D. Dissertation, Laboratoire de Thermique Appliquée et de Turbomachines, Swiss Federal Institute of Technology, Lausanne, Switzerland, 1996.

Körbächer, H., and Bölcs, A., "Steady-State and Time-Dependent Experimental Results of a NACA-3506 Cascade in an Annular Channel," ASME Paper 96-GT-334, 1996.

Lane, F., "System Mode Shapes in the Flutter of Compressor Blade Rows," Journal of the Aeronautical Sciences, pp. 54-66, Jan., 1956.

Panovsky, J., "Flutter of Aircraft Engine Turbine Blades," Ph.D. Dissertation, University of Cincinnati, 1997.

Panovsky, J., Nowinski, M., and Bölcs, A., "Flutter of Aircraft Engine Low Pressure Turbine Blades," The 8th International Symposium of Unsteady Aerodynamics and Aeroelasticity of Turbomachines, Stockholm, Sweden, Sept. 14-18, 1997.

Panovsky, J., and Kielb, R., "A Design Method to Prevent Low Pressure Turbine Blade Flutter," to be presented at the ASME Gas Turbine Conference and Exhibition, Stockholm, Sweden, June, 1998.

Zweifel, O., "The Spacing of Turbomachinery Blading, Especially with Large Angular Deflection," Brown Boveri Rev., Vol. 32, p.12, 1945. 